\title{
Dimensões da qualidade de vida no trabalho e justiça organizacional: um estudo com servidores públicos municipais
}

\author{
Dimensions of quality work life and organizational justice: a case with municipal public servants
}

\author{
Sandra Mara de Andrade ${ }^{1}$, Ana Cristina Limongi-França ${ }^{2}$ e Silvio Roberto Stefano ${ }^{3}$ \\ ${ }^{1}$ Universidade Estadual do Centro-Oeste, Brasil, Doutorado em Administração, e-mail: prof.sandraandrade@hotmail.com \\ ${ }^{2}$ Universidade de São Paulo, Brasil, Doutorado em Administração, e-mail: climongi@usp.br \\ ${ }^{3}$ Universidade Estadual do Centro-Oeste, Brasil, Doutorado em Administração, e-mail: professor-silvio@hotmail.com
}

Recebido em: 10/01/2019 - Revisado em: 20/03/2019 - Aprovado em: 06/05/2019 - Disponível em: 01/07/2019

\section{Resumo}

A promoção da Qualidade de Vida no Trabalho no Serviço Público pode ser vista sob três ângulos igualmente importantes: o do Bem-Estar dos próprios servidores, o da satisfação dos usuários cidadãos e o da eficiência e eficácia dos serviços prestados pelos órgãos públicos. O objetivo principal deste artigo é analisar a percepção de funcionários do serviço público municipal, a respeito de Qualidade e Vida no Trabalho sob a perspectiva do BPSO-99, bem como a percepção sobre Justiça organizacional. Caracteriza-se a pesquisa quanto aos seus diferentes aspectos como um estudo de campo, quantitativo e descritivo. A coleta de dados ocorreu por meio de pesquisa survey com os servidores públicos de uma prefeitura no interior do Paraná. Os dados foram tratados a partir de análises estatísticas univariadas e multivariadas. Os aspectos sociais foram os que apresentaram os piores índices de satisfação na percepção dos indivíduos pesquisados. Esse estudo contribui na proposição de um instrumento de diagnóstico da QVT na área pública municipal, sendo uma adaptação e validação do modelo BPSO-99, denominando-se BPSO-SP (Serviço Público), pois essa é uma lacuna encontrada na literatura e que poderá contribuir significativamente para novas pesquisas e avanços na gestão pública.

Palavras-chave: bem-estar organizacional, qualidade de vida, justiça, servidores públicos.

\begin{abstract}
The promotion of quality of life in the Public Service can be seen from three equally important angles: the welfare of the employees themselves, the satisfaction of citizen users and the efficiency and effectiveness of services provided by public agencies. The main objective of this paper is to analyze the perception of municipal public service employees regarding Quality and Work Life from the perspective of the BPSO-99, as well as the perception about
\end{abstract}


Organizational Justice. We characterize the research regarding its different aspects as a field study, quantitative and descriptive. The data collection was carried out through a survey survey with public servants of a city hall in the interior of Paraná. The data were treated from univariate and multivariate statistical analyzes. The social aspects were those that presented the worst satisfaction indexes in the perception of the individuals surveyed. This paper contributes to the proposal QWL diagnostic tool in the municipal public, being an adaptation and validation of the BPSO-99 model, denominated BPSO-SP (Public Service), since this a gap found in the literature and may contribute significantly to new research and advances in public management.

Keywords: organizational well-being, quality of life, justice, public servants.

\section{Introdução}

Diversas organizações tem esforços constantes de mudança que envolvem tecnologias, estratégias, processos e pessoas. Siqueira e Amaral (2006, p. 2) apontam que "o desafio para as organizações não está em sobreviver somente implantando novas políticas e estratégias de gestão, nem somente adquirindo tecnologias e equipamentos, mas, sobretudo, investindo no Bem-Estar dos seus recursos humanos". Neste sentido, modelos ou programas de Qualidade de Vida que observem os aspectos da interação entre o trabalhador e a organização são fundamentais para garantir a preservação da saúde e Bem-Estar do trabalhador.

A definição de qualidade de vida é tão abrangente quanto a dimensão do ser humano. A qualidade de vida, que tem um cunho holístico por considerar o indivíduo em todas as suas dimensões (LIMONGI-FRANÇA, 2011), é também um conceito multidimensional que visa à produtividade e à humanização do trabalho (FERNANDES, 1996; MEDEIROS, 2007; FERREIRA, et al, 2009; LIMONGI-FRANÇA, 2011; SANT'ANNA et al, 2011). Os estudos da qualidade de vida no trabalho - QVT seguiram uma abordagem sóciotécnica, impulsionada pela perspectiva de uma sociedade progressiva, baseada em satisfação dos colaboradores, saúde e segurança (LIMONGI-FRANÇA, 2011).

Estudos sobre QVT são desenvolvidos no setor privado, que buscam diagnosticar a Qualidade de Vida no Trabalho dos mais diversos profissionais, ou analisar e avaliar programas desenvolvidos pelas organizações, sendo que, no serviço público, a Gestão da Qualidade de Vida no Trabalho é um fenômeno embrionário. Apesar disso, a promoção da QVT no Serviço Público pode ser vista sob três ângulos igualmente importantes: o do BemEstar dos próprios servidores, o da satisfação dos usuários cidadãos e o da eficiência e eficácia dos serviços prestados pelos órgãos públicos (ALFENAS, 2013), visto que "a aplicação de QVT na administração pública é capaz de preencher uma lacuna [...] no nível de tratamento oferecido ao servidor público, relativa à valorização do seu trabalho e preocupação com seu bem-estar e o de sua família" (AMORIM, 2010, p. 38).

Dessa forma, o objetivo deste artigo é analisar a percepção de funcionários do serviço público municipal, a respeito de Qualidade e Vida no Trabalho sob a perspectiva do BPSO99, bem como a percepção sobre Justiça organizacional no serviço público.

Assim, entende-se que esse é um tema atual e de interesse da área de gestão de pessoas e administração pública, bem como da sociedade em geral. Pesquisar a Qualidade de Vida no Trabalho e a Justiça Organizacional no serviço público significa debruçar-se sobre uma questão fundamental, tendo em vista os impactos que podem representar a satisfação no 
trabalho, bem-estar organizacional e serviços públicos para a sociedade, que precisa dar sua contribuição na compreensão do tema.

Será apresentado nesse artigo o quadro teórico abordando a qualidade de vida no trabalho a justiça organizacional, a metodologia do estudo com os procedimentos da pesquisa, as análises dos resultados com as dimensões da qualidade de vida no trabalho e justiça organizacional na perspectiva dos servidores públicos municipais e as considerações finais. O estudo contribuiu na prática, pois foi possível entender como as dimensões da qualidade de vida no trabalho e justiça organizacional são percebidas pelos funcionários públicos municipais e as estratégicas organizacionais que poderiam ser desenvolvidas para isto. Para a temática contribui na proposição de um instrumento de diagnóstico da QVT na área pública municipal, sendo uma adaptação e validação do modelo BPSO-99 na área pública, denominando-se BPSO-SP (Serviço Público). A seguir apresenta-se o quadro teórico com os principais aspectos da qualidade de vida e qualidade de vida no trabalho e justiça organizacionais para o embasamento da temática.

\section{Quadro Teórico}

Foi desenvolvida uma bibliometria, analisando-se os anais do EnAnpad, principal congresso brasileiro de administração, no período temporal de 2004 a 2016, a fim de verificar como se apresentam as publicações sobre os temas estudados de modo específico no contexto brasileiro. Os dados dessa bibliometria, foram publicados nos anais do EnAnpad. Silva, Hayashi e Hayashi (2011) relatam que a bibliometria abrande dois tipos de estudos: os descritivos e os avaliativos. As pesquisas descritivas referem-se à produtividade obtida pela contagem de livros, periódicos e outros formatos de comunicação, enquanto que os estudos avaliativos estão relacionados ao uso da literatura por meio da contagem de referências e citações em trabalhos de pesquisa. Esse estudo envolveu um estudo descritivo e também avaliativo, pois realiza contagem de publicações e o número de citações em outras publicações.

Os mesmos autores, destacam ainda que as leis de dispersão de Bradford e de produtividade científica de Lotka serem consideradas como marcos na bibliometria, na verdade as pesquisas bibliométricas iniciaram em finais dos anos 1960. Já a partir década de 1990, a bibliometria se tornou uma ferramenta padrão da política científica (SILVA, HAYASHI, HAYASHI, 2011).

A revisão bibliométrica da literatura utilizou como palavras chave "Qualidade de Vida no Trabalho" e "Justiça Organizacional". A busca realizada ocorreu em: título, resumo (abstract), palavra-chave (keywords). Foram identificadas 49 publicações, no decorrer de 10 anos. A análise dos artigos selecionados foi realizada considerando o período temporal, a autoria, os principais autores citados e as temáticas relacionadas aos dois conceitos. Verificouse constância em relação às publicações sobre QVT, já em relação à Justiça Organizacional, pode-se inferir que é um campo ainda não consolidado na área da Administração, visto que, em alguns períodos, nenhum artigo foi publicado. Outro indicativo de que Justiça Organizacional é uma temática ainda em desenvolvimento é que, dos 14 artigos, 3 foram desenvolvidos por um único autor, o que corresponde a mais de $20 \%$ das publicações.

Dentre os autores internacionais mais citados nos trabalhos pesquisados, há as obras de Walton, Westley e Hackman e Oldham. Geralmente são usados os modelos desses autores para o diagnóstico de QVT nas organizações. A autora nacional mais citada é Limongi- 
França, em virtude de apresentar o modelo biopsicossocial organizacional para Gestão de Qualidade de Vida no Trabalho; seguida por Fernandes, Morin, e Sant'anna e Kilimnik.

Dentre as obras sobre Justiça Organizacional, a mais citada é a de Colquitt (2001), visto que propõe uma escala para mensurar justiça de forma multidimensional. Outro autor citado é Jerald Greenberg (1993) considerado o pai do termo Justiça Organizacional. Dentre os autores que publicam em língua portuguesa, destacam-se Rego (2002), Assmar et al. (2005) e Gomide Jr (2002). O que causou estranheza foi a obra de Rawls, "A theory of justice", considerada uma obra relevante quando se trata do desenvolvimento do campo da Teoria da Justiça, ter sido referenciada em apenas 3 trabalhos.

Verificou-se que os artigos não tratam das temáticas (Qualidade de Vida no Trabalho e Justiça Organizacional) de forma associada, ou seja, das relações existentes entre elas, mas alguns temas se apresentam associados as duas temáticas como: comprometimento e satisfação. Já a aproximação de Justiça Organizacional com assuntos relacionados à QVT ocorrem a partir de estudos que envolvem Burnout, Percepção da Saúde e Bem-Estar. Os resultados dessa bibliometria fortalecem a justificativa de que há uma lacuna nas publicações brasileiras sobre a temática, visto que são incipientes os estudos que relacionam os construtos Qualidade de Vida no Trabalho e Justiça Organizacional. Os resultados também inspiraram na escolha dos modelos-base para esta pesquisa: o BPSO, de Limongi-França (1996) e escala de Justiça Organizacional, de Colquitt (2001), pois os dois são as maiores referências nos estudos brasileiros, cada qual em sua temática, em relação às escalas ou modelos para mensuração e análise das temáticas. A seguir apresenta-se o Quadro Teórico do tema de pesquisa.

\subsection{Qualidade de Vida no Trabalho}

Qualidade é um conceito milenar; no século XX, ganhou força como um conceito estratégico nas organizações em decorrência da competitividade. Alguns autores ganharam destaque nesse contexto, influenciando significativamente a história da qualidade, como: Deming (1982), Crosby (1984), Taguchi (1986), Juran (1988) e Ishikawa (1990), a partir do desenvolvimento teorias ou de ferramentas para a qualidade. Deming (1982) foi o precursor da filosofia da Gestão da Qualidade Total, a qual resulta do reconhecimento de que as melhorias da qualidade e da gestão levam a um aumento da produtividade. No Serviço Público, a perspectiva da qualidade total passou a ser preconizada a partir do movimento gerencialista, e preconizado pela Nova Administração Pública.

O grupo de qualidade de vida da Organização Mundial da Saúde - OMS a define como "A percepção do indivíduo de sua posição na vida, no contexto da cultura e sistema de valores em que vive e em relação a suas expectativas, seus padrões e suas preocupações." (WHOQOL, 1995, p. 1405). “Essa definição tem sido uma das mais citadas na literatura sobre o assunto, tratando a QV como uma construção subjetiva (percepção do indivíduo), multidimensional e composta por dimensões positivas, como a mobilidade, e negativas, como a dor." (ALFENAS, 2013, p. 6).

Limongi-França (2011) descreve que a definição de qualidade de vida é tão abrangente quanto a dimensão do ser humano; para a autora, qualidade de vida tem um cunho holístico por considerar o indivíduo em todas as suas dimensões e seus estudos são desenvolvidos por diversas áreas do conhecimento. Assim, mesmo em países em que tais estudos estão 
bastante desenvolvidos, como nos Estados Unidos (quality of working life - QWL), Canadá, França (Qualité de la Vieau Travail - QVT) e, de modo especial, Suécia, em que esta linha de pesquisas alcançou considerável desenvolvimento, não há consenso na definição (FERNANDES, 1996).

A base para esta pesquisa foi o modelo teórico biopsicossocial e organizacional (BPSO-96) desenvolvido por Limongi-França, conforme Quadro 1.

\section{Quadro 1: Dimensões de QVT - BPSO}

\begin{tabular}{|c|c|c|}
\hline Dimensão & Esforço da empresa & Satisfação do funcionário \\
\hline $\begin{array}{l}\text { Biológica } \\
\text { constitucionais herdadas e congênitas, } \\
\text { incluindo os diferentes órgãos e } \\
\text { sistemas que promovem o } \\
\text { funcionamento do corpo, inclusive a } \\
\text { resistência e a vulnerabilidade do } \\
\text { corpo; }\end{array}$ & $\begin{array}{l}\text { - Semana de prevenção de } \\
\text { acidentes } \\
\text { - Prevenção de riscos - PPRA } \\
\text { - Ginástica } \\
\text {-Controle médico - PCSMO } \\
\text { - Atuação da CIPA }\end{array}$ & $\begin{array}{l}\text {-Qualidade das SIPAT's } \\
\text { - Controle de riscos ergonômicos e } \\
\text { ambientais } \\
\text { - Oportunidade de realizar ginástica } \\
\text { - Estado de saúde dos colegas e } \\
\text { superiores } \\
\text { - Atuação da CIPA }\end{array}$ \\
\hline $\begin{array}{l}\text { Psicológica }- \text { corresponde } \\
\text { processos afetivos, emocionais } \\
\text { intelectuais, e conscientes } \\
\text { inconscientes, caracterizando a } \\
\text { personalidade, a vida mental, o afeto e } \\
\text { o jeito de se relacionar com as pessoas } \\
\text { e com o mundo que as rodeia. }\end{array}$ & $\begin{array}{l}\text { - Critérios de recrutamento e } \\
\text { seleção } \\
\text { - Ferramentas de avaliação de } \\
\text { desempenho } \\
\text { - Gestão do clima } \\
\text { organizacional } \\
\text { - Plano de carreira } \\
\text { - Administração de salários } \\
\text { - Respeito à vida pessoal } \\
\text { - Estabilidade } \\
\end{array}$ & $\begin{array}{l}\text { - Confiança no recrutamento e } \\
\text { seleção } \\
\text { - Formas de avaliação de } \\
\text { desempenho } \\
\text { - Clima de camaradagem } \\
\text { - Oportunidade de carreira } \\
\text { - Satisfação com o salário } \\
\text { - Ausência de interferência na vida } \\
\text { pessoal } \\
\text { - Segurança no emprego }\end{array}$ \\
\hline $\begin{array}{l}\text { Social - relativa à incorporação e às } \\
\text { influências dos valores, das crenças e } \\
\text { as expectativas das pessoas com as } \\
\text { quais se convive, dos grupos sociais e } \\
\text { das diferentes comunidades com as } \\
\text { quais o indivíduo entra em contato } \\
\text { durante sua vida. }\end{array}$ & $\begin{array}{l}\text { - Convênios comerciais } \\
\text { - Atividades de lazer } \\
\text { - Atendimento aos filhos } \\
\text { - Financiamento de cursos } \\
\text { externos }\end{array}$ & $\begin{array}{l}\text { - Qualidade dos convênios } \\
\text { comerciais } \\
\text { - Oportunidade de distração } \\
\text { - Atendimento aos filhos (creche, } \\
\text { escola) } \\
\text { - Financiamento de cursos externos }\end{array}$ \\
\hline $\begin{array}{l}\text { Organizacional - aspectos específicos } \\
\text { do local onde as relações de produção } \\
\text { ocorrem. }\end{array}$ & $\begin{array}{l}\text { - Endomarketing } \\
\text { - Programa de T\&D } \\
\text { - Implantação de processos e } \\
\text { tecnologias } \\
\text { - Gestão participativa } \\
\text { - Redução da burocracia } \\
\text { - Atendimento às rotinas de } \\
\text { pessoal }\end{array}$ & $\begin{array}{l}\text { - Imagem da empresa } \\
\text { - Oportunidade de T\&D } \\
\text { - Melhorias nos processos e } \\
\text { tecnologias } \\
\text { - Participação em comitês } \\
\text { - Qualidade dos processos } \\
\text { administrativos } \\
\text { - Atendimento a registro, férias, } \\
\text { pagamento etc }\end{array}$ \\
\hline
\end{tabular}

Fonte: Elaborado a partir de Limongi-França (1999, p. 240).

As quatro dimensões apresentadas no Quadro 1: biológica, psicológica, social e organizacional de QVT-BPSO são relacionados a diversos esforços da organização e apresenta efeitos na satisfação dos funcionários.

Quanto ao comprometimento com a carreira, os diretores apresentam um aspecto afetivo de significância e identificação com a carreira.

\subsection{Justiça Organizacional}

Os esforços para explicar o impacto da Justiça sobre o funcionamento organizacional eficaz ocorreram com os estudos sobre Justiça Organizacional. De acordo com Colquitt (2001), Justiça Organizacional é comumente descrita a partir de dois tipos de percepções subjetivas: Justiça Distributiva e justiça procedimental. Gomide Jr (2002) descreve que se identificam na literatura duas correntes teóricas: uma que trata da Justiça na perspectiva de Aristóteles, como um fenômeno unidimensional e outra que trata o construto como 
multidimensional. Colquitt (2001) descreve, ainda, que foi Greenberg (1993) quem trouxe uma nova perspectiva para os debates de Justiça, sugerindo uma estrutura de quatro fatores para a Justiça Organizacional. Essa estrutura é apresentada no Quadro 2 a seguir:

Quadro 2: Dimensões de Justiça Organizacional e principais estudiosos

\begin{tabular}{|l|l|l|}
\hline $\begin{array}{l}\text { Dimensões de } \\
\text { Justiça }\end{array}$ & Caracterização & Principais Estudiosos \\
\hline Distributiva & $\begin{array}{l}\text { Primeira dimensão estudada pelos cientistas } \\
\text { organizacionais. Seu foco é no conteúdo, ou seja, na justiça } \\
\text { dos fins alcançados ou obtidos, sejam eles positivos ou } \\
\text { negativos (ex: salários, lucros, resultados de avaliações de } \\
\text { desempenho ou sanções). }\end{array}$ & Adams (1965) \\
\hline Procedimental & $\begin{array}{l}\text { Seu foco é o processo, ou seja, na justiça dos meios usados } \\
\text { para alcançar os fins (ex: procedimentos usados para } \\
\text { movimentação salarial e bonificações, processos } \\
\text { disciplinares, sistemas de avaliação de desempenho, } \\
\text { processos de recrutamento e seleção). }\end{array}$ & $\begin{array}{l}\text { Thibat e Walker (1975; } \\
1978) ; \text { Lind e Tyler (1988) }\end{array}$ \\
\hline $\begin{array}{l}\text { Interacional } \\
\text { Interpessoal }\end{array}$ & $\begin{array}{l}\text { Trata da qualidade da interação entre empregados e } \\
\text { superiores ou decisores, reflete o grau de adoção de um } \\
\text { tratamento digno e respeitador para com os funcionários } \\
\text { (ex:o gestor age com dignidade e respeito?). }\end{array}$ & $\begin{array}{l}\text { Bies e Moag (1986); Tyler } \\
\text { (1993), e Rego (2000d) }\end{array}$ \\
\hline $\begin{array}{l}\text { Interacional } \\
\text { Social/ } \\
\text { Informacional }\end{array}$ & $\begin{array}{l}\text { Reflete a qualidade da interação com os gestores/decisores, } \\
\text { sobre o grau em que o superior informa, explica e justifica } \\
\text { as decisões que afetam as pessoas (ex: oferece justificações } \\
\text { aos indivíduos afetados pelas decisões?). }\end{array}$ & $\begin{array}{l}\text { Greenberg e } \\
\text { (1990); }\end{array}$ \\
\hline
\end{tabular}

Fonte: Adaptado de Rego (2002, p.216) e Demo (2010, p.24).

Ainda sobre os conceitos das dimensões, “Justiça Distributiva se refere ao conteúdo das distribuições, à justiça dos resultados alcançados, e a justiça processual, diz respeito à justiça dos procedimentos adotados para determinar as distribuições" (ASSMAR et al, 2005, p. 444). O conceito de Justiça procedimental, cujo foco são os processos, foi desenvolvido por Thibaut e Walker (1975) e aprimorado por Leventhal (1980), o qual introduziu o conceito no contexto organizacional (COLQUITT, 2001).

A Justiça Organizacional como um aspecto da Qualidade de Vida no Trabalho, no serviço público, pode ser verificada no campo das teorias de serviço público, pois quando tratam da reforma de estado e suas influências na gestão de pessoas, Nogueira e Santana (2000, p.2) destacam que,

Nesse contexto de discussão, não se pode deixar de considerar a atualidade do princípio do mérito que, como expressão da igualdade de oportunidades, rege o acesso a cargos públicos e constitui o fundamento das prerrogativas da burocracia. No entanto, noções mais contemporâneas e complexas de justiça distributiva vêm procedendo a uma recontextualização desse princípio, o que o torna de valor relativo.

Dessa forma, os autores demonstram que na Nova Gestão Pública a dimensão justiça distributiva ou organizacional faz-se relevante no contexto da administração pública (municipal, estadual ou federal).

Pelo campo da Gestão da Qualidade de Vida no Trabalho, identificou-se os estudos de Reddy e Reddy (2010), que tratam da Justiça Administrativa como uma proposta emergente, ou uma técnica, para melhoria da qualidade de vida. Os autores definem Justiça Administrativa como os princípios de justiça: "justo e de qualidade deve ser aplicado em processo disciplinar, queixa, procedimentos, promoções, transferências, atribuição de trabalho, licença, etc." Essa definição de justiça assemelha-se muito ao de Justiça Distributiva, utilizada por autores brasileiros e portugueses. A seguir apresenta-se os procedimentos metodológicos da pesquisa. 


\section{Procedimentos Metodológicos}

A presente pesquisa caracteriza-se quanto aos seus diferentes aspectos como um estudo de campo, quantitativo e descritivo. Quanto à tipologia e objetivo, esta pesquisa é descritiva, pois busca identificar quais as situações ou eventos estão expressos em uma determinada população. Quanto à dimensão tempo, caracteriza-se como um estudo de corte transversal, ou seja, os dados são coletados num único momento (EASTERBY-SMITH et al., 1999).

A unidade de análise deste estudo consiste de 4.060 servidores públicos municipais lotados em 17 secretarias municipais que fazem parte do Poder Executivo Municipal de um município no interior do Estado do Paraná.

A escala utilizada para os blocos de variáveis do BPSO-96 e JOSP foi a do tipo Likert (1932), de sete pontos, conforme a legenda. De acordo com Dalmoro e Vieira (2013, p. 165-166), a escala de 7 pontos apresenta uma série de vantagens, como: limite da habilidade humana de distinção; permite melhor discriminação; ganho de consistência interna e confiabilidade; ponto neutro (decorrente das escalas ímpares); boa discriminação da covariância; bom ajuste às estatísticas multivariadas, sendo esse o principal motivo por se optar por essa amplitude de escala, já que a modelagem de equações estruturais é uma técnica multivariada. Sua desvantagem é haver uma grande quantidade de casos (respondentes) para inferências.

Pré-teste - o questionário, em suas diferentes etapas de elaboração, foi pré-testado exaustivamente. Inicialmente, a escala do BSPO-JOSP foi aplicada aos 84 servidores do Poder Legislativo do mesmo município e se verificou que muitos itens não estavam de acordo com a realidade e com a linguagem adequada ao tipo de público, visto que diversos ficaram sem resposta, devido aos questionamentos e comentários dos respondentes (em meados de 2014). Posteriormente, foram realizadas as operacionalizações de construtos para o serviço público e o novo questionário foi submetido à validação de construtos por 3 especialistas da na área de Gestão de pessoas no serviço público. Segundo Dunn et al (1994), os construtos devem ser definidos com base na literatura em conjunto com o entendimento do pesquisador sobre estes. O questionário ainda passou pela validação de 10 participantes das entrevistas e focusgroup e foi novamente pré-testado com 15 estudantes do mestrado profissional em administração, dos quais dois são funcionários públicos.

Esse estudo apresenta a adaptação e validação do modelo BPSO-99 na área pública, pois foi importante para a criação do instrumento de diagnóstico e pesquisa denominado BPSO-SP (Serviço Público), sendo essa uma lacuna encontrada na literatura do tema.

O processo de amostragem adotado nesta pesquisa obedeceu a amostragem probabilística, estratificada por Secretaria Municipal. A principal característica desse modelo de amostragem é poder ser submetida a tratamento estatístico, que permite compensar erros amostrais e outros aspectos relevantes para a representatividade e significância da amostra (MARCONI, LAKATOS, 2002), ou seja, os resultados podem ser generalizados para a população estudada, isto é, para a unidade de análise do estudo.

Considerando o contingente da população da Unidade (4.060 funcionários públicos, lotados em 17 secretarias municipais) e aplicada a fórmula para cálculo da amostra para população finita, foi necessária uma amostra composta por 352 respondentes.

Para a extratificação considerou-se que 352 respondentes correspondem a $9 \%$ da população. Dessa forma, para fins de arredondamento, foi realizado sorteio aleatório, com o uso do Excel, de 10\% da população para cada secretaria, considerando as possíveis ausências 
dos indivíduos ou a recusa em responder à pesquisa. Para as secretarias com menos de 10 funcionários, sorteou-se um funcionário para responder a pesquisa.

A análise dos dados quantitativos coletados foi desenvolvida utilizando análise univariada e multivariada, conforme os objetivos a serem atingidos. O processamento dos dados foi feito utilizando o software SPSS para as análises descritivas, as analises são detalhadas no item Analise das Dimensões BPSO - JOSP em Relação aos dados sócio demográficos dos servidores públicos. A seguir apresenta-se as análises das dimensões da pesquisa.

\section{Análises dos Resultados}

\subsection{Campo de Pesquisa}

A prefeitura investigada está localizada na mesorregião centro-sul do estado do Paraná, em um polo populacional de mais de 500 mil habitantes, o município possui uma população estimada em 180.334 pelo IBGE (2018). A prefeitura possui um total de 4.263 servidores em Agosto de 2018 (GUARAPUAVA, 2018).

Em relação ao perfil dos respondentes, todos são servidores públicos municipais, vinculados ao Poder Executivo municipal, ou seja, à prefeitura do município. Dos 379 questionários válidos, 345 funcionários estão vinculados ao município por aprovação em concurso estatutário, o que representa 91\% dos respondentes. Contratados em comissão foram 23 servidores que participaram da pesquisa, o que correspondea 6,1\% dos questionários respondidos, e 11 participantes da pesquisa, 2,9\%, estão vinculados ao município via regime CLT - Consolidação das Leis do Trabalho.

Em relação ao sexo dos servidores respondentes, 313 são mulheres e 66 participantes são homens, o que corresponde respectivamente $83 \%$ e $17 \%$, o que está em acordo com dados da PNAD - pesquisa nacional por amostra de domicílios, de que as mulheres já são maioria entre a população economicamente ativa (PEA), desde 2012 (PNAD, 2013) e também corresponde à realidade da instituição pesquisada, uma vez que as duas maiores secretarias em termos de número de servidores são a Secretaria de Educação e de Saúde, sendo que a composição do quadro de servidores dessas secretarias é predominantemente feminino.

\subsection{Analise das Dimensões BPSO - JOSP em Relação aos Dados Sócio Demográficos dos Servidores Públicos}

Este item contempla a análise de como se apresentam as relações entre as dimensões e os dados sócio demográficos dos servidores públicos, a fim de verificar se existem diferença de percepção em relação aos constructos, analisando as características dos respondentes da pesquisa. Foram analisadas as seguintes características: sexo, idade, estado civil, escolaridade, tempo de instituição, salário, dependentes e se acredita ou não que a pesquisa pode ser utilizada para ações de Qualidade de Vida na instituição. Para essas analises foram excluídos os dados NT (não existe na instituição, ou não se aplica), ou seja, o zero foi substituído por vazio. Dessa forma, o número $(\mathrm{N})$ de pesquisados analisados varia, não sendo a totalidade de 379, dessa forma, é dado o (N) nas tabelas a seguir: 


\section{* Sexo}

A análise das dimensões em relação ao sexo foi realizada por meio do teste $t$ para amostras independentes, esse teste se dá por meio do comparativo das médias de duas amostras, no caso, homens e mulheres, por meio do teste de hipóteses. Considera-se H0: não existe diferença significativa entre as médias de respostas de homens e mulheres para as dimensões (BPSO, JOSP) e H1: existe diferença significativa entre as médias de respostas de homens e mulheres para as dimensões (BPSO, JOSP). Considerando a tabela do teste $t$, recomenda-se neste caso, rejeitar a hipótese 0 ou nula quando $t \geq 1,64$, considerando uma

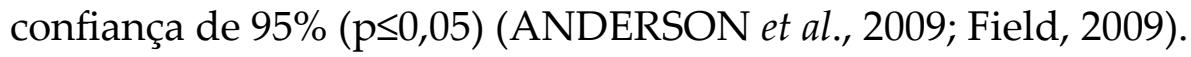

Tabela 1: Relação Sexo - BPSO - JOSP

\begin{tabular}{l|c|c|c|c|c|c}
\hline \multirow{2}{*}{ CONSTRUCTOS } & \multicolumn{2}{|c|}{ Masculino } & \multicolumn{2}{|c|}{ Feminino } & \multirow{2}{*}{$\mathrm{T}$} & \multirow{2}{*}{$\mathrm{p}$} \\
\cline { 2 - 6 } & $\mathrm{N}$ & \multicolumn{1}{|c}{ Média } & $\mathrm{N}$ & \multicolumn{1}{c}{ Média } & & \\
\hline BIOLÓGICOS & 65 & 3,81 & 284 & 3,49 & 1,657 & 0,09 \\
\hline PSICOLÓGICOS & 66 & 4,77 & 313 & 4,47 & 1,939 & 0,05 \\
\hline SOCIAIS & 63 & 3,95 & 279 & 3,84 &, 524 & 0,60 \\
\hline ORGANIZACIONAIS & 65 & 4,00 & 310 & 3,79 & 1,158 & 0,24 \\
\hline JOSP & 66 & 4,56 & 311 & 4,18 & 2,085 & 0,03 \\
\hline
\end{tabular}

Fonte: dados da pesquisa

Verifica-se, a partir da tabela 1 , que se rejeitou (pois $p \leq 0,05$ e $t \geq 1,64$ ) não existe diferença significativa entre as médias de respostas de homens e mulheres para as dimensões Psicológico $(p=0,05)$ e Justiça Organizacional $(p=0,03)$. Assumiu-se então que há diferença significativa nas médias em relação ao sexo, sendo que o sexo masculino apresentou médias significativamente superiores que o sexo feminino, nos constructos Psicológico $(4,77)$ e Justiça Organizacional $(4,56)$. Verificou-se ainda que o sexo masculino apresentou valores de medias superiores para todas as dimensões, entretanto, para as demais a diferença não é significativa.

Em relação à Justiça, estudos correlatos geralmente se concentram em um aspecto da Justiça Organizacional, Paz (1992) aponta que estudos a respeito da percepção de Justiça Distributiva que buscam identificar diferenças de percepção em relação a sexo são pouco conclusivos, nesse caso, em que o constructo Justiça Organizacional no Serviço Público é formado pelos quatro tipos de justiça (procedimental, distributiva, interacional e informacional) verificou-se que o sexo masculino percebe mais Justiça, isso pode se dar, nessa amostra em razão de haver diferença significativa de salário, sendo a renda percebida pelos homens maior que a percebida pelas mulheres.

\section{* Idade}

A relação da idade dos servidores com os constructos da pesquisa foi analisada a partir do teste de correlação de Pearson. Dentro dos parâmetros descritos por Field (2009) 0,1 indica pequenas correlações 0,3 indica correlação de efeito médio e 0,5 efeito grande de correlação. Identificou-se correlação da média de idade, 42 anos, com Justiça Organizacional, respectivamente, que indica a que a correlação é significativa, com $96 \%$ de confiança. Entretanto, a magnitude das correlações foi baixa $(r=0,11)$ evidenciando quase nenhuma influência prática da idade nas escalas, portanto, os resultados não foram conclusivos.

\section{* Estado Civil}

As análises de estado civil com os constructos da pesquisa foram desenvolvidas a partir do Método da Análise de Variância (ANOVA), e não foi verificada diferença significativa em relação a nenhum dos constructos, e não foram identificados outros estudos sobre 
qualidade de vida no trabalho ou justiça organizacional que apresentassem essa análise para se estabelecer comparativos.

\section{* Escolaridade}

A análise de escolaridade com os constructos foi desenvolvida pelo método da Análise de Variância (ANOVA). Os parâmetros a serem utilizados a fim identificar se há diferença significativa foram a tabela de $(\mathrm{F})$, nesse caso se apresentam 3 graus de liberdade no numerador, sendo assim, o parâmetro para se identificar se há diferença significativa é F $\infty$ $=\mathrm{F}>2,60$ (Oliveira e Stahl, 2013) considerando uma confiança de 95\% ( $\leq \leq 0,05)$ (ANDERSON et al., 2009; FIELD, 2009).

Para essa análise foram reunidos, em um único grupo os respondentes que marcaram a opção especialização, mestrado/doutorado, visto que um único pesquisado estava enquadrado na opção mestrado/doutorado.

Tabela 2: Relação Escolaridade - BPSO - JOSP

\begin{tabular}{l|c|c|c|c|c|c|c}
\hline Constructos / Médias & $\begin{array}{l}\text { Funda- } \\
\text { mental }\end{array}$ & Médio & Superior & $\begin{array}{l}\text { Especia- } \\
\text { lização }\end{array}$ & N & F & p. \\
\hline BIOLÓGICOS & 3,47 & 3,42 & 3,70 & 3,60 & 349 & $\begin{array}{l}\mathrm{F}(3 ; 345)= \\
, 771\end{array}$ & 0,51 \\
\hline PSICOLÓGICOS & 4,34 & 4,52 & 4,56 & 4,60 & 378 & $\begin{array}{l}\mathrm{F}(3 ; 374)= \\
, 648\end{array}$ & 0,58 \\
\hline SOCIAIS & 4,07 & 3,95 & 3,89 & 3,70 & 341 & $\begin{array}{l}\mathrm{F}(3 ; 337) \\
=, 845\end{array}$ & 0,47 \\
\hline ORGANIZACIONAIS & 3,46 & 3,71 & 3,96 & 3,99 & 374 & $\begin{array}{l}\mathrm{F}(3 ; 370)= \\
2,602\end{array}$ & 0,05 \\
\hline JOSP & 4,05 & 4,09 & 4,43 & 4,34 & 376 & $\begin{array}{l}\mathrm{F}(3 ; 372)= \\
1,625\end{array}$ & 0,18 \\
\hline
\end{tabular}

Fonte: Dados da pesquisa.

A partir da tabela 2, verifica-se que há diferença significativa nas médias no constructo organizacionais $(p=0,05)$. Para identificar em que grupos estavam as diferenças foi utilizado post hoc de Bonferroni, que conforme já descrito na metodologia, consiste em comparações em pares planejadas para comparar todas as diferentes combinações dos grupos testados (FIELD, 2009).

Verificou-se diferença significativa com $(p=0,05)$ entre o grupo ensino fundamental com ensino superior e especialização. Pelo teste post hoc de Bonferroni temos que o grupo Ensino fundamental teve valores significativamente inferiores aos grupos Ensino superior $(\mathrm{p}=0,03)$ e grupo Especialização (mestrado e doutorado) $(\mathrm{p}=0,02)$, assim verifica-se que as pessoas com maior escolaridade percebem melhor os aspectos organizacionais. Não foram identificados estudos que relacionassem escolaridade com os aspectos organizacionais para fazer comparativos.

* Salario

A análise de escolaridade em relação aos constructos foi desenvolvida pelo método da Análise de Variância (ANOVA). Os parâmetros a serem utilizados a fim identificar se há diferença significativa foram a tabela de $(\mathrm{F})$, nesse caso se apresentam 3 graus de liberdade no numerador, sendo assim, o parâmetro para se identificar se há diferença significativa é $\mathrm{F}_{\infty}=\mathrm{F}>2,60$ (OLIVEIRA, STAHL, 2013) considerando uma confiança de 95\% $(\mathrm{p}<0,05)$ (ANDERSON et al., 2009; FIELD, 2009). Os dados das médias são apresentados na tabela 3. 
Tabela 3: Relação Escolaridade - BPSO - JOSP

\begin{tabular}{|c|c|c|c|c|c|c|c|}
\hline \multirow[t]{2}{*}{ CONSTRUCTOS } & \multicolumn{4}{|c|}{ Médias das faixas salariais } & \multirow[b]{2}{*}{$\mathrm{N}$} & \multirow[b]{2}{*}{$\mathrm{F}$} & \multirow[b]{2}{*}{$\mathrm{p}$} \\
\hline & $\begin{array}{c}\text { Até } \\
788,00\end{array}$ & $\begin{array}{c}\mathrm{De} \\
788,00 \mathrm{a} \\
1576,00\end{array}$ & $\begin{array}{c}\text { De } \\
1576,00 \mathrm{a} \\
4728,00\end{array}$ & $\begin{array}{c}\text { Acima } \\
\text { de } \\
4728,00\end{array}$ & & & \\
\hline BIOLÓGICOS & 3,06 & 3,62 & 3,48 & 3,77 & 349 & $\begin{array}{l}\mathrm{F}(3 ; 345)= \\
2,54\end{array}$ & 0,05 \\
\hline PSICOLÓGICOS & 3,94 & 4,59 & 4,61 & 4,62 & 379 & $\begin{array}{l}\mathrm{F}(3 ; 375)= \\
4,73\end{array}$ & 0,30 \\
\hline SOCIAIS & 4,02 & 4,01 & 3,72 & 3,69 & 342 & $\begin{array}{l}\mathrm{F}(3 ; 338)= \\
1,15\end{array}$ & 0,32 \\
\hline ORGANIZACIONAIS & 3,22 & 3,81 & 3,91 & 4,08 & 375 & $\begin{array}{l}\mathrm{F}(3 ; 371)= \\
4,38\end{array}$ & 0,005 \\
\hline JOSP & 3,72 & 4,29 & 4,29 & 4,38 & 377 & $\begin{array}{l}\mathrm{F}(3 ; 373)= \\
2,67\end{array}$ & 0,04 \\
\hline
\end{tabular}

Fonte: Dados da pesquisa.

Verifica-se que para um nível de significância de $95 \%(\mathrm{p}<0,05)$ se apresentam diferenças significativas, em relação à renda percebida com os aspectos biológicos, organizacionais, justiça organizacional e bem-estar. Para identificar em que grupos estavam as diferenças foi utilizado post hoc de Bonferroni, que conforme já descrito na metodologia, consiste em comparações em pares planejadas para comparar todas as diferentes combinações dos grupos testados (Field, 2009). E identificou-se no aspecto biológico $(\mathrm{p}=0,05)$, pelo que o grupo até $\mathrm{R} \$ 788$ apresentou valores significativamente inferiores aos grupos $\mathrm{R} \$ 788$ a 1.576 $(\mathrm{p}=0,02)$ e grupo acima de $\mathrm{R} \$ 4.728(\mathrm{p}=0,009)$.

A diferença verificada nos aspectos organizacionais, também evidenciou que o grupo até $\mathrm{R} \$ 788$ apresentou valores significativamente inferiores a todos os outros 3 grupos para $\mathrm{R} \$ 788$ a $1.576(\mathrm{p}=0,008)$; para $\mathrm{R} \$ 1576$ a $4.728(\mathrm{p}=0,003)$ e para acima $\mathrm{R} \$ 4.728(\mathrm{p}<0,001)$, o que indica significância de mais de $95 \%(\mathrm{p}<0,05)$ em todos os casos. Justiça organizacional também melhor percebida pelos grupos com maiores remunerações, para o grupo até $\mathrm{R} \$$ 788 os valores se apresentaram significativamente inferiores a todos os outros 3 grupos $(\mathrm{p}=$ 0,01 para $R \$ 788$ a $1.576 ; p=0,02$ para $R \$ 1576$ a 4.728 e $p=0,008$ para acima $R \$ 4.728$ ). Diante do apresentado, conclui-se que as pessoas com maior remuneração percebem de forma mais positiva as dimensões biológicas e organizacionais, assim como a justiça organizacional no serviço público.

\section{* Tempo na Instituição}

Tempo na instituição em relação aos constructos da pesquisa foi analisado a pelo teste de correlação de Pearson. O tempo médio na instituição foi de 10 anos e a única correlação observada foi com os aspectos sociais $(r=-0,10 ; p=0,05)$, o que significa uma correlação negativa significativa, por exemplo, a medida que o tempo de casa aumenta a percepção sobre os aspectos sociais diminui. Entretanto, como a magnitude da correlação é baixa baixas quase nenhuma influência prática nas escalas se evidencia. Os resultados aqui encontrados para justiça, o fato de não haver correlação com tempo de serviço, são opostos aos Santos e Odelius (2008) que identificaram a variável tempo de trabalho na área apresenta-se como preditora da justiça organizacional.

\section{* Dependentes}

A percepção das pessoas com e sem dependentes em relação às dimensões BPSO, JOSP, BESP foram testadas por meio da análise do teste $t$. Observada a tabela do teste $t$, considera-se neste caso que há diferença significativa, quando $t \geq 1,64$, com uma confiança de $95 \%(p<0,05)$ (ANDERSON et al., 2009; FIELD, 2009). A tabela 4 apresenta os dados do teste $\mathrm{t}$ realizado. 

com servidores públicos municipais

Tabela 4: Relação Dependentes -BPSO - JOSP

\begin{tabular}{l|c|c|c|c|c|c}
\hline \multirow{2}{*}{ CONSTRUCTOS } & \multicolumn{2}{|c|}{ Tem dependentes } & \multicolumn{2}{|c|}{ Não tem dependentes } & \multirow{2}{*}{$\mathrm{t}$} & \multirow{2}{*}{$\mathrm{p}$} \\
\cline { 2 - 6 } & $\mathrm{N}$ & $\mathrm{Média}$ & $\mathrm{N}$ & Média & & \\
\hline BIOLÓGICOS & 238 & 3,61 & 111 & 3,42 & $-1,202$ & 0,23 \\
\hline PSICOLÓGICOS & 259 & 4,56 & 120 & 4,46 &,- 771 & 0,44 \\
\hline SOCIAIS & 232 & 3,86 & 110 & 3,86 &, 006 & 0,99 \\
\hline ORGANIZACIONAIS & 257 & 3,89 & 118 & 3,68 & $-1,411$ & 0,15 \\
\hline JOSP & 257 & 4,33 & 120 & 4,04 & $-1,950$ & 0,05 \\
\hline
\end{tabular}

Fonte: Dados da pesquisa.

Foram encontradas diferenças significativas apenas para justiça organizacional ( $p$ $=0,05$ ), sendo que os servidores com dependentes apresentam valores significativamente superior do que aqueles que não tem dependentes. Não foram identificados outros estudos que analisaram essa relação para comparações.

* Acredita ou não que a pesquisa pode ser utilizada para ações de qualidade de vida na instituição

A relação dos constructos (BPSO - JOSP) com a questão acredita ou não que a pesquisa pode ser utilizada para ações de qualidade de vida na instituição foi analisada por meio do teste $t$, com significância de 95\% (p<0,05) (ANDERSON et al., 2009; FIELD, 2009).

Tabela 5: Relação Acredita -BPSO - JOSP

\begin{tabular}{l|c|c|c|c|c|c}
\hline \multirow{2}{*}{ CONSTRUCTOS } & \multicolumn{2}{|c|}{ Acredita } & \multicolumn{2}{c|}{ Não acredita } & \multirow{2}{*}{$\mathrm{t}$} & \multirow{2}{*}{$\mathrm{p}$} \\
\cline { 2 - 5 } & $\mathrm{N}$ & Média & $\mathrm{N}$ & Média & & \\
\hline BIOLÓGICOS & 94 & 3,56 & 255 & 3,53 &,- 182 & 0,85 \\
\hline PSICOLÓGICOS & 111 & 4,52 & 268 & 4,55 &, 269 & 0,78 \\
\hline SOCIAIS & 95 & 3,94 & 247 & 3,67 & $-1,431$ & 0,15 \\
\hline ORGANIZACIONAIS & 108 & 3,89 & 267 & 3,67 & $-1,639$ & 0,10 \\
\hline JOSP & 109 & 4,36 & 268 & 3,95 & $-2,960$ & 0,003 \\
\hline
\end{tabular}

Fonte: Dados da pesquisa.

A partir dos dados da tabela 5, verifica-se que os servidores que entendem que a pesquisa pode ser utilizada para ações de qualidade de vida na instituição apresentaram melhor percepção dos que os que não acreditam em relação à justiça organizacional no serviço público $(\mathrm{p}=0,003)$.

Os estudos internacionais (RAI, 2015; REDDY, REDDY, 2010) descrevem o crescente interesse nas temáticas Qualidade de Vida no Trabalho e justiça, bem como o estudo das relações entre esses constructos nos mais diferentes tipos de organizações. Os autores ainda identificaram relações positivas entre Justiça Organizacional e Qualidade de Vida no Trabalho. Já na literatura nacional, observa-se uma lacuna quando se trata das relações entre QVT e Justiça Organizacional. Dessa forma, a contribuição principal desse estudo para a teoria é oferecer uma explicação para a relação entre Justiça Organizacional e QVT no Brasil, considerando como unidade de análise o serviço público, sendo que a relação aqui proposta foi a de antecedência de justiça em relação à QVT. A seguir apresenta-se as considerações finais do estudo.

\section{Considerações Finais}

O presente artigo teve como objetivo analisar a percepção de funcionários do serviço público municipal, a respeito de Qualidade e Vida no Trabalho sob a perspectiva do BPSO99, bem como a percepção sobre Justiça organizacional no serviço público. Constatou-se que, embora obteve-se relações não significativas entre os aspectos biológicos e sociais com 
Bem-Estar podem ser decorrentes da percepção baixa de satisfação desses dois fatores, o que é condizente com estudos anteriores de Limongi-França (2009b). A análise desse resultado não pode ser desvinculada da pesquisa qualitativa, na qual se verifica que os aspectos como paternalismo referentes à cultura do serviço público interferem na percepção da Qualidade de Vida no Trabalho, conforme identificou Schirrmeister (2006). Esse resultado também reflete a necessidade da organização institucionalizar práticas que priorizem o atendimento aos problemas de saúde do servidor e divulgar práticas institucionalizadas, como o ambulatório multiprofissional, o qual não é reconhecida por funcionários de várias secretarias. Outro indicador que destaca a necessidade de práticas voltadas para a saúde é que 52,5\% dos servidores utilizaram remédios para dores específicas nos últimos três meses.

As relações positivas de Bem-Estar no Serviço Público com os aspectos psicológico e organizacional do BPSO eram evidenciadas em outros estudos (LIMONGI-FRANÇA, 2009, PACHECO, 2011; DESSEN, PAZ, 2009). A relação do indivíduo com a instituição é influenciada pela remuneração. No construto Psicológico, a manifestação da relação do servidor público com a instituição ocorreu a partir do variável salário, adequado ao trabalho realizado, e no fator organizacional ficou evidente a necessidade de melhorias nos processos de trabalho, que se apresentou também na pesquisa qualitativa.

As analises qualitativas do estudo contribuíram muito além da operacionalização dos constructos, pois permitiram verificar a importância de aspectos culturais, como a burocracia; ele não só influencia na implementação de práticas voltadas para a Qualidade de Vida no Trabalho em organizações públicas, mas também prejudica a QVT dos servidores, à medida que há morosidade na compra de equipamentos, manutenção de aspectos ergonômicos do ambiente de trabalho, além de impactar os aspectos referentes à valorização do servidor.

Os resultados do estudo refletem a necessidade que as instituições públicas têm em possuir estratégias bem definidas de Gestão da Qualidade de Vida no Trabalho, pois outro aspecto cultural do serviço público, o paternalismo, faz com que o servidor coloque toda a responsabilidade pela sua qualidade de vida na instituição, o qual não identifica que a Qualidade de Vida no Trabalho depende não só de um programa organizacional institucionalizado de gestão de QVT, mas também de ações individuais.

O estudo pretende contribuir com a percepção dos servidores públicos quanto as dimensões da qualidade de vida no trabalho e justiça organizacional, analisando a visão destes em relação às estratégias de QVT na perspectiva da gestão pública municipal.

Para a gestão pública, fornece um conjunto de indicadores que podem facilitar a implementação de práticas de gestão de Qualidade de Vida no Trabalho voltadas para o Bem-Estar no Serviço Público, por meio de uma perspectiva biopsicossocial e organizacional. Além disso, demonstra que justiça é relevante para a Qualidade de Vida no Trabalho.

Propõe-se um instrumento de diagnóstico da QVT na área pública municipal, sendo uma adaptação e validação do modelo BPSO-99 na área pública, denominando-se BPSO-SP (Serviço Público), pois essa é uma lacuna encontrada na literatura e que poderá contribuir significativamente para novas pesquisas e avanços na área pública.

As limitações deste estudo apresentam-se, à medida que a unidade de análise são servidores públicos municipais locais, o que inviabiliza a generalização dos resultados para outros contextos, como esferas públicas estaduais e federais. 


\section{Referências}

LFENAS, R. A. S. Qualidade de vida no trabalho na administração pública: concepções de gestores e avaliação por subordinados. Dissertação de Mestrado. Centro Universitário das Faculdades Associadas de Ensino - FAE. São João da Boa Vista, 2013.

AMORIM, T. G. F. N. Qualidade de vida no trabalho: preocupação também para servidores públicos? Revista Eletrônica de Ciência Administrativa - RECADM. v.9, n.1: maio, 2010. Disponível em: http://revistas.facecla.com.br/ index.php/recadm/article/view/357/465. Acesso em: 25.11.2018.

ANDERSON, D. R. et al. Estatística aplicada à administração e economia. 2. ed. São Paulo: Cengage Learning, 2009.

ASSMAR, E. M L.et al. Justiça Organizacional: uma revisão crítica da literatura. Psicologia: Reflexão e Crítica. v.18, n.3, 443-453, 2005.

COLQUITT, J.A. On the Dimensionality of Organizational Justice: A construct validation of a Measure. Journal of Applied Psychology. v.86, n.3, 368-400, 2001.

DALMORO, M.; Vieira, K. M. Dilemas Na Construção De Escalas Tipo Likert: O Número De Itens E A Disposição Influenciam Nos Resultados? Revista Gestão Organizacional - RGO. v.6, (ed. Especial), 161-174, 2013.

DESSEN, M. C.; PAZ, M. G. T. Indicadores de Bem-estar Pessoal nas Organizações: em busca de uma avaliação. In: Anais do XXXIII Encontro da ANPAD - EnANPAD. 1. São Paulo, p. 1-112, 2009.

DUNN, S. C.et al. Latent variables in business logistics research: Scale development and validation. Journal of Business logistics. v.15, n.2: 145-172, 1994.

EASTERBY-SMITH, M.et al. Pesquisa gerencial em administração: um guia para monografia, dissertações, pesquisas internas e trabalhos em consultoria. São Paulo: Pioneira, 1999.

FERNANDES, E. C. Qualidade de vida no trabalho: como medir para melhorar. Salvador: Casa da Qualidade, 1996.

FERREIRA, M. C. et al. Gestão de Qualidade de Vida no Trabalho (QVT) no Serviço Público Federal: O Descompasso entre Problemas e Práticas Gerenciais. Psicologia: Teoria e Pesquisa. v.25, n.3: 319-327, 2009.

FIELD, A. Descobrindo a Estatística utilizando o SPSS. 2 ed. Artmed, 2009.

GOMIDE JR., S. Justiça nas Organizações In: SIQUEIRA M.M.M. et al(Org). Cidadania, Justiça e Cultura nas Organizações: estudos psicossociais. São Bernardo do Campo: Universidade Metodista de São Paulo, 2002, 105-162.

GREENBERG, J. Organizational justice: yesterday, today and tomorrow. Journal of Management. v.16, n.1, 399-432, 1993. 
GUARAPUAVA.Portal da Transparência.Quadrodefuncionários públicosmunicipais.http:// transparencia.guarapuava.pr.gov.br:2000/pronimtb/index.asp?acao=4\&item=2Acesso em $13 / 12 / 2018$.

IBGE. Instituto Brasileiro de Geografia e Estatística. https://cidades.ibge.gov.br/brasil/pr/ guarapuava/panorama Acesso em 13/12/2018.

LEVENTHAL, G. S. What should be done with equity theory? New approaches to the study of fairness in social relationships. In: GERGEN, K. et al (Org.). Social exchange: Advances in theory and research. New York: Plenum Press, 1980.

LIKERT, R. A technique for the measurement of attitudes. Archives of Psychology. v.140: 44-53, 1932.

LIMONGI-FRANÇA, A. C. Indicadores empresariais de qualidade de vida no trabalho: esforço empresarial e satisfação dos empregados no ambiente de manufaturas com certificação ISO 9000. Tese de Doutorado. Faculdade de Economia, Administração e Contabilidade da Universidade de São Paulo - FEA-USP. São Paulo, 1996.

LIMONGI-FRANÇA, A. C. Promoção de saúde e qualidade de vida no trabalho: o desafio da gestão integrada. In: ROSSI, A. M. et al (Org). Stress e Qualidade de vida no trabalho: o positivo e o negativo. São Paulo: Atlas, 2009a.

LIMONGI-FRANÇA, A. C. Relatório da Consulta no XYZ Sobre Expectativas, Ações e Programas De Qualidade De Vida No Trabalho. Relatório Técnico, 2009b.

LIMONGI-FRANÇA, A. C. Qualidade de Vida no Trabalho-QVT: conceitos e práticas nas empresas da sociedade pós-industrial. 2. ed. São Paulo: Atlas, 2011.

LIMONGI-FRANÇA, A. C. RG-QVT - Núcleo de Gestão de Qualidade de Vida no Trabalho, 2017. Disponível em: http://www.g-qvt.com.br/. Acesso em: 22.02.2018.

MARCONI, M. A.; LAKATOS, E. M. Técnicas de pesquisa. 5. ed. São Paulo: Atlas, 2002.

MEDEIROS, J. P. Qualidade de Vida no Trabalho da EMATER-RN: validação de um instrumento síntese de pesquisa e diagnóstico. Dissertação de mestrado. Universidade Federal do Rio Grande do Norte, Natal, 2007.

NOGUEIRA, R.N; SANTANA, J. P. Gestão de Recursos Humanos e Reformas do Setor Público: Tendências e Pressupostos de uma Nova Abordagem. Documento elaborado sob encomenda do Programa de Desenvolvimento de Recursos Humanos da Organização Pan-Americana da Saúde. Workshop on Global Health Workforce Strategy., Annecy: Organização Mundial da Saúde, 2000.

OLIVEIRA, F. M.; STAHL, N. S. P. Análise de variância aplicada em pesquisas sociais. Linkania. v.7, n.1, 2013. 
PACHECO, V. A. Qualidade de vida no trabalho, bem-estar e mal-estar sobre a ótica de uma agencia reguladora no Brasil. Dissertação de Mestrado. Instituto de Psicologia da Universidade de Brasília, 2011.

RAI, G. S. Organizational Justice and Quality of Working Life: A Road That Leads to a Virtuous Organization. Journal of Social Service Research. v.41, n.2: 269-294, 2015.

REDDY. M. L.; REDDY. P. M. Quality of work life of employees: emerging dimensions. Asian Journal of Management Research. v.1, n.2: 827-839, 2010.

REGO, A. Comprometimento afectivo dos membros organizacionais: o papel das percepções de justiça. Revista de Administração Contemporânea. v.6, n.2: 209-241, 2002.

SANTOS, A. R.; ODELIUS, C. C. Percepção de Justiça Organizacional de Sistemas de Remuneração em Organizações Públicas. Revista Alcance Eletrônica, v.15, n.02: 226-242, 2008.

SCHIRRMEISTER, R. Qualidade de vida no trabalho e comprometimento organizacional: um estudo em equipes multicontratuais em um instituto de pesquisa tecnológica.Dissertação de mestrado. Faculdade de Economia, Administração e Contabilidade da Universidade de São Paulo - FEA-USP. São Paulo, 2006.

SILVA, M. R.; HAYASHI, C. R. M.; HAYASHI, M. C. P. I. Análise bibliométrica e cintométrica: desafios para especialistas que atuam no campo. R. Ci. Inf. e Doc., Ribeirão Preto, v.2, n.1: 110-129, 2011.

SIQUEIRA, M. M. M.; AMARAL, D. J. Relações entre Estrutura Organizacional e Bem-Estar Psicológico. REA - Revista Eletrônica de Administração. v.5, n.1, 2006.

THE WHOQOL GROUP. The World Health Organization Quality of Life Assessment: position paper from the World Health Organization. Social Science \& Medicine. v.41, n.1: 1403-1410, 1995.

THIBAUT, J. W.; WALKER, L. Procedural justice: a psychological analyses. New York: Erlbaum, Hillsdale, 1975. 